\title{
Dual-stage gain-clamped erbium-doped fiber amplifier with fiber Bragg grating
}

\begin{abstract}
We demonstrate a dual-stage gain-clamped erbiumdoped fiber amplifier. The first-stage amplifier consists of a short length of erbium-doped fiber to produce low noise figures. The second-stage is constructed from a counter-propagating ringlaser, in which the signals and the lasing wavelength propagate in the opposite direction. The lasing wavelength is selected via a reflective-type of fiber Bragg grating. The gain-clamping mechanism can be adjusted by either changing the fiber Bragg grating reflectivity or center wavelength. The noise figure penalty is about $1.5 \mathrm{~dB}$ for the gain-clamping value from $11 \mathrm{~dB}$ to $20.5 \mathrm{~dB}$. (@) 2008 by Astro Ltd., Published exclusively by WILEY-VCH Verlag GmbH \& Co. KGaA)
\end{abstract}

Keyword: erbium, optical amplifier, gain-clamped 